\title{
Cross-Sectional Examination of Crystallinity of Carbon Fibers by Transmission Electron Microscopy
}

\author{
Z.P. Luo, Jiwoong Sue, ${ }^{* *}$ Ozden Ochoa,* and Andreas Holzenburg ${ }^{* *}$ \\ "Microscopy and Imaging Center and " Department of Biology, BSBW, Texas A\&M University, \\ College Station, TX 77843-2257 \\ ${ }^{* *}$ Dept. of Mechanical Engineering, Texas A\&M University, College Station, TX 77843-3123
}

Carbon fibers are attractive light structural materials especially for the aerospace industry due to their distinguished advantages such as low density, high specific tensile modulus and strength. [1] Within the fiber, although the graphite structure layers primarily align along the fiber axis, the stacking of these layers along the transverse direction produces complicated domain structures with different size and orientation, and the properties of the fiber are influenced by the crystallinity status. The aim of this work is to examine the crystallinity in the cross-section of the carbon fibers by transmission electron microscopy (TEM).

The carbon fibers (T1000 and T300 series) presented here were part of a broader research program sponsored by NASA (Grant NAG 8-1882). Fibers with different heat treatment histories were embedded in epoxy and thin-sectioned along the cross-sectional axis of the fiber. Thin sections were collected and deposited onto grids coated with holey carbon films. Specimens were observed in a JEOL 2010 at $200 \mathrm{kV}$.

Fig. 1 shows an example of a cross-section of a non-heat-treated as-received T1000 series fiber. The ring electron diffraction pattern from the core area of the fiber (upper right) displays maxima corresponding to the $d_{002}$ spacing of the graphite structure. [2] In the lattice image (lower right), the graphite layer fringes are visible but show various orientations. The diffraction pattern from the skin area (upper left) displays a broadened (002) reflection, [3] indicating that this skin area may be more ordered than the core area.

Fig.2 shows the T1000 fiber being subjected to three thermal cycles from room temperature to 800 ${ }^{\circ} \mathrm{C}$. On the left side, the core area still has less graphite ordering similar to that shown in Fig. 1. However, the diffraction pattern (upper right) from the skin area clearly reveals a broad (002) reflection, and in the lattice image (lower right), more aligned parallel graphite fringes are visible in this region. From this it appears that heat treatment produces graphite ordering only in the skin area but not in the core area.

However, for fibers which were heat treated by the manufacturer at high temperatures, the crystallinity changes not only in the skin but also the core area. Fig. 3 shows a comparison of the lattice images as well as the corresponding diffraction patterns (inserts) from the core areas of the original T300 series sample (Fig. 3a) and a sample after heating to approximately $2500{ }^{\circ} \mathrm{C}$ (Fig. 3b). After such a treatment, the graphite layers in the core area appear to be realigned (Fig. 3b). These observations are consistent with measurements of the coefficient of thermal expansion (CTE), which reveal that heat-treated samples with more graphic ordering have lower CTE values [4]

\section{References}

[1] D.D.L. Chung, Carbon fiber composites, Butterworth-Heinemann, Boston, 1994.

[2] M. Guigon, A. Oberlin, and G. Desarmot, Fibre Sci. Technol. 20 (1984) 55.

[3] S.C. Bennett, and D.J. Johnson, Carbon 17 (1978) 25.

[4] The support provided by Dr. Michael Effinger of NASA Marshall Research Center is gratefully acknowledged. 


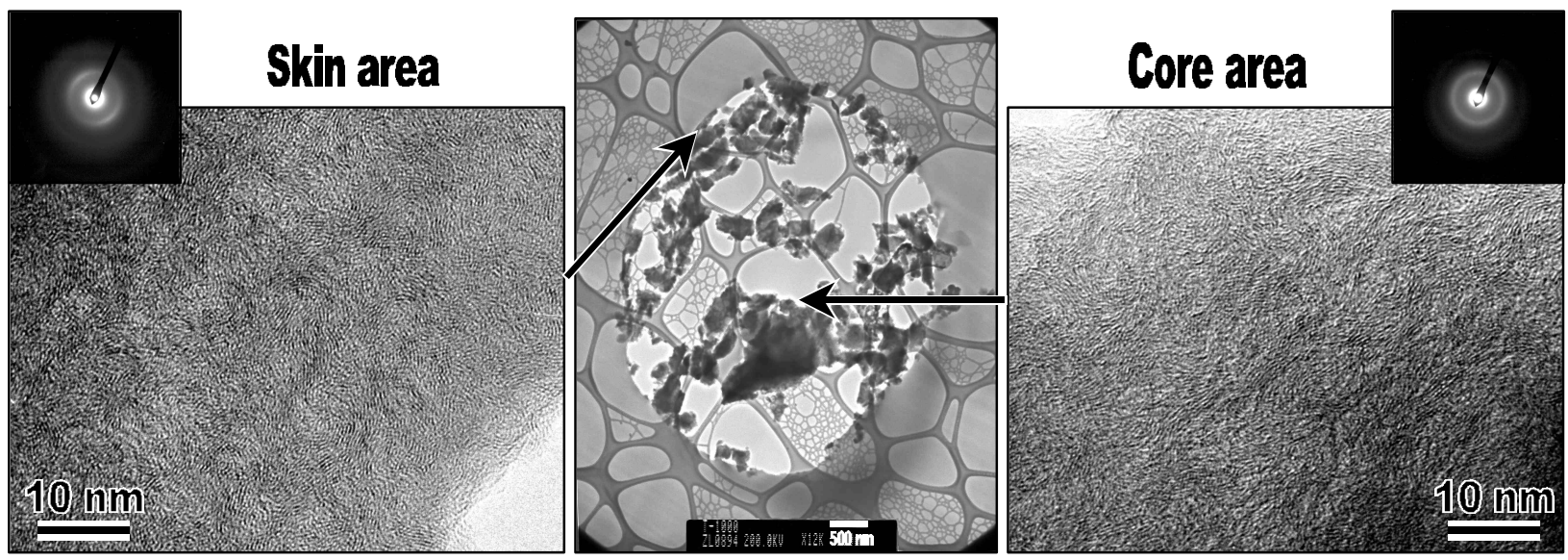

FIG. 1. Cross sections of a fiber (overview, center) showing the structure of the skin area (left) and core area (right).
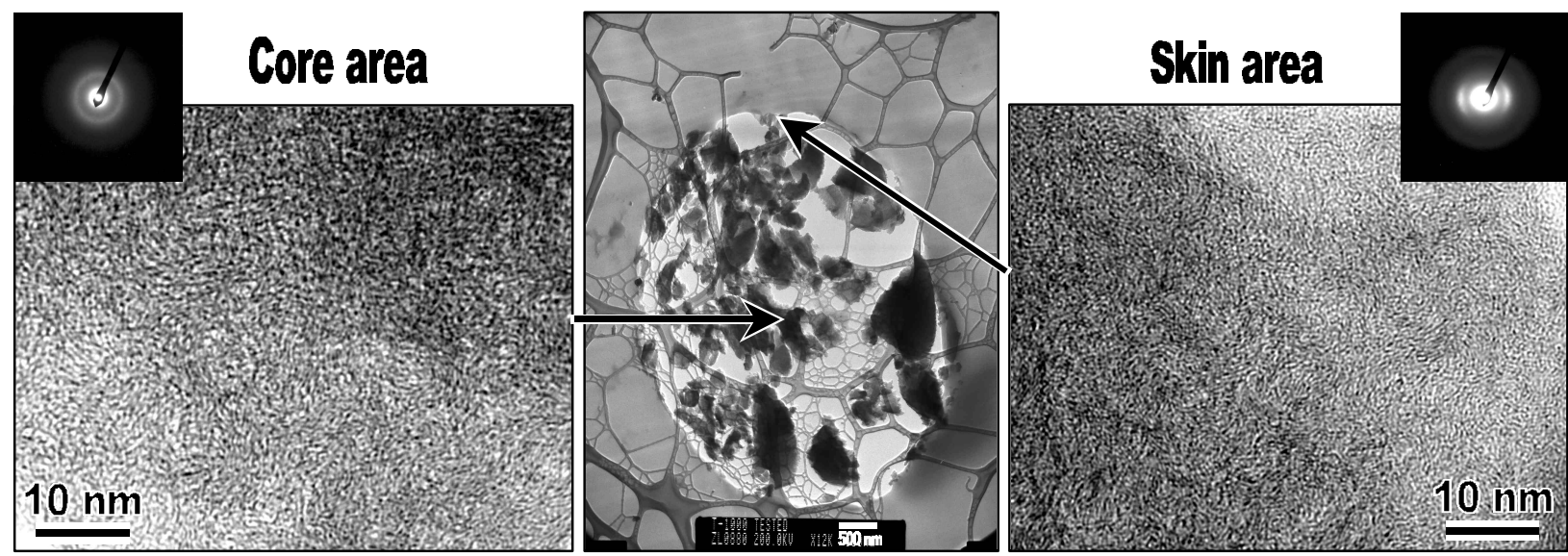

FIG. 2. Cross sections of a fiber after heating to $800{ }^{\circ} \mathrm{C}$ (overview, center) showing the structure of the core area (left) and skin area (right).
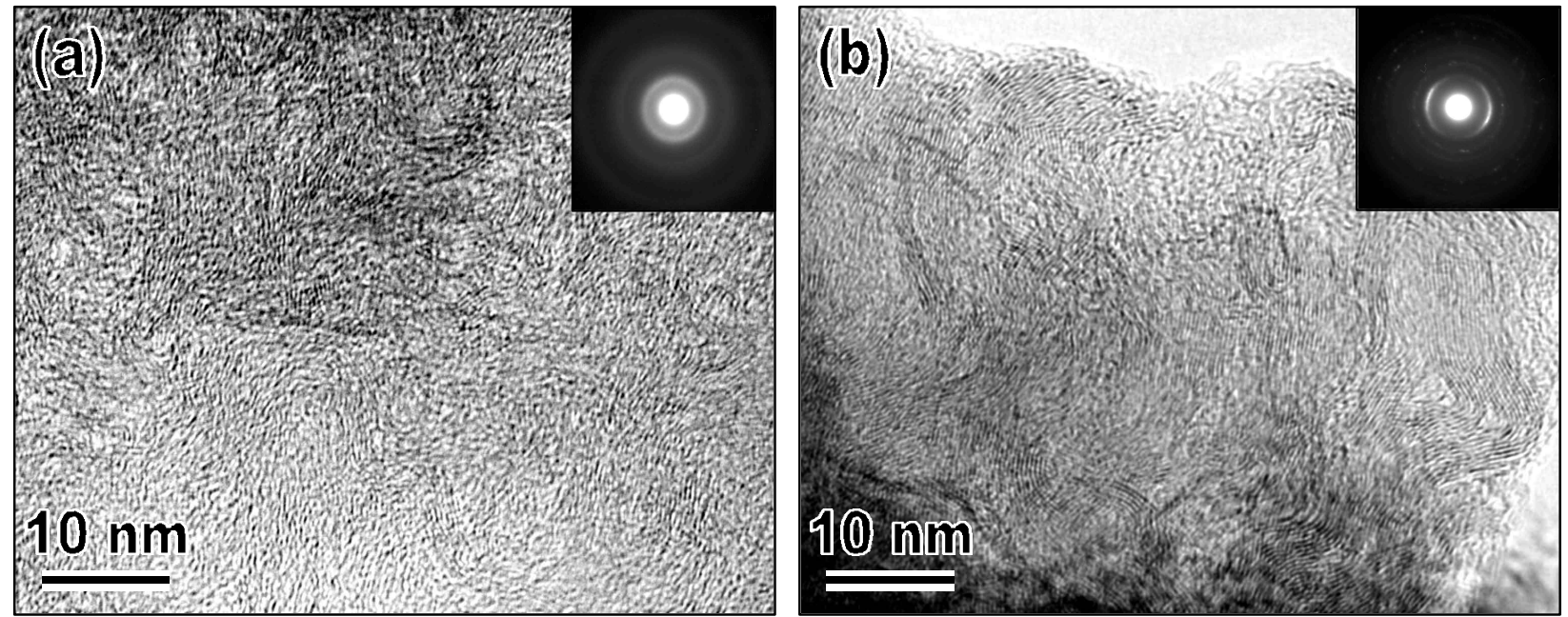

FIG. 3. Comparison of the core areas of the original (a) and heat-treated sample (b). 